\title{
Recursos financieros y humanos en el servicio comunitario de una universidad pública venezolana ${ }^{1}$
}

\section{Financial and human resources in the community service of a venezuelan public university}

DOI: http://dx.doi.org/10.17981/econcuc.38.2.2017.02

Recibido: mar 1 /2017 Devuelto para revisión: mar 18/17 Aceptado: ago 14/2017

\author{
María Gabriela Díaz ${ }^{2}$ \\ José Manuel Gutiérrez ${ }^{3}$ \\ Jenny Romero ${ }^{4}$ \\ Mayra Alejandra Villalobos ${ }^{5}$
}

\begin{abstract}
Resumen
Este trabajo tiene como objetivo identificar los recursos financieros y humanos del servicio comunitario del Núcleo Humanístico de la Universidad del Zulia, Venezuela. La investigación es descriptiva, utilizando la entrevista para la recolección de información. Los resultados ponen en evidencia que los recursos financieros provienen del presupuesto ordinario de la universidad, que con el tiempo se hicieron insuficientes, llevando a tutores y estudiantes a autofinanciar los insumos requeridos. En cuanto al recurso humano, se tiene que los actores del proceso se muestran identificados con la actividad, pero la escasez de recursos financieros podría influir en la desmotivación de estos. Las instancias pertinentes deben garantizar los medios necesarios para desarrollar esta política institucional.
\end{abstract}

Palabras clave: recursos financieros; recursos humanos; servicio comunitario; universidad.

\begin{abstract}
The purpose of this research is to identify the financial and human resources of the Humanistic Nucleus' community service of the Universidad del Zulia, Venezuela. This is a descriptive research, where the interview was used to data colection. The results show that the financial resources come from the ordinary budget of the university, which over time became insufficient, leading tutors and students to self-finance the required inputs. Regardind the human resource, the actors in the process are identified with the activity, but the scarcity of financial resources could influence their demotivation. The pertinent instances must guarantee the necessary means to develop this institutional policy.
\end{abstract}

Keywords: Financial resourses; human resourses; communite service; university.

${ }^{1}$ Este artículo es resultado del trabajo de investigación: Política institucional para la implementación del servicio comunitario del las facultades del Núcleo Humanístico de la Universidad del Zulia.

${ }^{2}$ Licenciada en Administración. Especialista en Docencia para la Educación Superior. Magister Scientiarum en Gerencia Pública. Docente - Investigadora adscrita al Centro de Estudios de la Empresa de la Facultad de Ciencias Económicas y Sociales de la Universidad del Zulia. Maracaibo, Venezuela. Correo: gabydn80@yahoo.es

${ }^{3}$ Economista. Personal Docente en Formación del Instituto de Investigaciones "Econ. Dionisio Carruyo" de la Facultad de Ciencias Económicas y Sociales de la Universidad del Zulia. Maracaibo, Venezuela. Correo: jm_gutiérrez@fces.luz.edu.ve

${ }^{4}$ Doctora en Ciencias Humanas. Magister Scientiarum en Gerencia de Empresas. Economista. Docente - Investigadora adscrita a la Universidad de la Costa (Barranquilla, Colombia) y a la Facultad de Ciencias Económicas y Sociales de la Universidad del Zulia (Maracaibo, Venezuela). Correo: jennyfrb@yahoo.com

${ }^{5}$ Economista. Magister Scientiarum en Gerencia de Empresas, Mención Gerencia Financiera. Docente - Investigadora adscrita al Instituto de Investigaciones "Econ. Dionisio Carruyo" de la Facultad de Ciencias Económicas y Sociales de la Universidad del Zulia. Maracaibo, Venezuela. Correo: mayravillalobos2001@gmail.com 


\section{Introducción}

La universidad se considera como un motor generador de cambios de conducta, de convivencia ciudadana, con gran impacto para el logro del desarrollo sustentable de nuestras comunidades. Sin embargo, en los últimos años, la institución universitaria, ente social generador de conocimientos y formador de capital humano, experimenta un fuerte cuestionamiento en relación con su desconexión con el entorno (Tünnerman, 2000). Situación que ha incidido en la poca pertinencia social, pertinencia que trasciende las demandas de la economía, del sector laboral o profesional, hasta cubrir las necesidades y carencias de la sociedad en la que está inmersa.

Es así como, organizaciones multilaterales, como la UNESCO y la misma universidad, han propiciado la discusión sobre el rol social de las universidades en el mundo. Ahora bien, este repensar sobre la universidad surge no sólo por los cambios internacionales o la importancia que se le dé a este tema fuera del país, sino también, por la aparición de nuevas leyes en el escenario jurídico venezolano que implican la participación de la universidad y su gente como actores clave.

De esta manera, en el año 2005, se aprueba en Venezuela la Ley de Servicio Comunitario del Estudiante de Educación Superior (LSCEES), según Gaceta Oficial No 38.272 del 14 de septiembre del mencionado año. Con esta ley se da un giro a la vinculación de la universidad con el entorno, objetivo central de la extensión universitaria de acuerdo con la Ley de Universidades (1970). Presentándose como una oportunidad para impulsar la relación directa universidad-sociedad al incorporar de manera masiva al estudiante a las actividades extramuros de acercamiento a la realidad que viven las comunidades.

La LSCEES, enmarcada en el texto constitucional (1999) y la Ley de Universidades (1970), pretende que la universidad asuma un rol de mayor impacto en la sociedad, vinculándose en la solución de los problemas que afectan a las comunidades desde el punto de vista económico, científico, tecnológico, cultural, social, político y educativo, entre otros. El objetivo central de esta ley es acercar al estudiante a su entorno para generar respuestas efectivas en las comunidades a partir de los proyectos desarrollados por las Instituciones de Educación Superior (IES). Asimismo, el servicio comunitario es una forma de retribución del estudiante a la sociedad (Pérez et al., 2014), siendo uno de los espacios para la aplicación de los conocimientos adquiridos en sus años de estudios.

De acuerdo con Hernández (2006) y Urdaneta, Godoy y Barreto (2014), la LSCEES busca fomentar la ética, la participación ciudadana y la reciprocidad social para lograr el desarrollo sostenible de las comunidades a través de la metodología aprendizaje-servicio, referida al servicio solidario desarrollado por los estudiantes destinado a cubrir necesidades reales de una comunidad, planificado institucionalmente en forma integrada con el currículo en función del aprendizaje de los estudiantes.

La Universidad del Zulia (LUZ), en el marco de la LSCEES, aprobó el Reglamento de Servicio Comunitario del Estudiante de Pregrado (2006), estableciendo las directrices para el desarrollo de esta política institucional concebida bajo la perspectiva del currículo integral, insertándose en los planes de estudio como eje transversal. Para el año 2012, este reglamento pasa por unas modificaciones en función de la dinámica académica-administrativa de estas actividades y se deroga la primera versión.

A más de una década de gestión del servicio comunitario y en atención a los recursos financieros y humanos de esta política institucional cabe preguntarse: ¿cuentan las facultades con recursos financieros y humanos suficientes para seguir llevando a cabo esta actividad? ¿Cómo ha sido la respuesta 
de los distintos actores vinculados con la ejecución del servicio? Bajo estas consideraciones, la presente investigación tiene el objetivo de identificar los recursos financieros y humanos en el servicio comunitario de las facultades del Núcleo Humanístico de LUZ: Ciencias Económicas y Sociales (FCES), Ciencias Jurídicas y Políticas (FCJP) y Humanidades y Educación (FHE).

Metodológicamente, la investigación se inscribe dentro del tipo de estudio descriptivo con un diseño no experimental de campo. Para la recolección de información se empleó la técnica de la entrevista mediante el uso de una guía semiestructurada aplicada a los cuatro (4) responsables de las instancias ejecutoras del servicio comunitario en LUZ para el momento (abril, 2017): Coordinador Central del Servicio Comunitario y los coordinadores de servicio comunitario de las tres (3) facultades del Núcleo Humanístico seleccionadas para el estudio.

\section{Extensión universitaria: vinculación universidad-entorno}

Las IES desempeñan un rol de suma importancia en la formación de recursos humanos del más alto nivel y en la creación, desarrollo, transferencia y adaptación de tecnología; de manera que lo que ellas hacen para responder adecuadamente a los requerimientos de la sociedad moderna se constituye en un imperativo estratégico para el desarrollo nacional.

En Venezuela, de acuerdo con las disposiciones fundamentales establecidas en el Título I de la Ley de Universidades (1970), el recinto universitario es el espacio destinado a realizar las funciones básicas de docencia, investigación y extensión. Funciones derivadas de la constante búsqueda de nuevos conocimientos, su transferencia y aplicación. Tradicionalmente, se ha hecho mayor énfasis en las actividades relacionadas con la función de docencia, por cuanto constituye la práctica cotidiana del quehacer académico, sin embargo, en los últimos años, la investigación ha experimentado un crecimiento notorio debido a su revalorización como plataforma para la innovación y el desarrollo de nuevos saberes. Por su parte, la función de extensión se ha mantenido aislada en actividades que no alcanzan a comprometer integralmente a la institución universitaria con su entorno (Tünnerman, 2003).

En el marco de la Primera Conferencia Latinoamericana de Extensión Universitaria llevada a cabo en Santiago de Chile en el año 1957, se adoptó un concepto de extensión universitaria enfocado en tres aspectos a destacar: 1) por su naturaleza, es misión y función orientadora de la universidad; 2) por su contenido y procedimientos, se fundamenta en el conjunto de estudios y actividades filosóficas, científicas, artísticas y técnicas necesarias para relacionarse y conocer el medio social; y 3) por sus finalidades, se centra en proyectar dinámica y coordinadamente la cultura, así como vincular la institución con la sociedad (Ramos, 2007, p. 62).

Posteriormente, en la Segunda Conferencia Latinoamericana de Difusión Cultural y Extensión Universitaria llevada a cabo en México, en 1972, el concepto de extensión universitaria fue reconfigurado y quedó expresado en términos de la interacción entre la universidad y los demás componentes del cuerpo social, a través del cual ésta asume y cumple su compromiso de participación en el proceso social de creación de la cultura y de la liberación y transformación radical de la comunidad nacional (Tünnerman, 2000, p. 188). Esta definición asumida por la Segunda Conferencia propone una postura más amplia, ya que proporciona a la universidad el compromiso de la interacción social, que sólo se logra desarrollando la extensión universitaria de una manera consecuencial y engranada con su principal objetivo, sustentado en la construcción del cambio social. 
En este orden de ideas, Torres y Cano (1997) conceptualizan la extensión universitaria como un proceso integral sistemático, continuo y dialógico entre los miembros de la comunidad intra y extrauniversitaria, con el propósito de elevar la creación y transformación permanente de la cultura (saberes, conocimientos, habilidades, aptitudes y valores, arte, tecnología, experiencias, entre otros), a fin de promover con equidad la calidad de vida de la sociedad.

Asimismo, Malagón (2002) observa la extensión como una función inherente al propio proceso de aprendizaje del estudiante, el cual podrá aprender a través de la acción desde la localidad y región, constituyéndose, además, en un promotor de cambio social. En esta perspectiva, la extensión ha de ser un proceso esencialmente de liberación en el cual el hombre se convierte en sujeto responsable de la historia y partícipe en ella de una forma creadora.

En el marco de las concepciones señaladas, cuando se habla de extensión universitaria, se puede entender como un proceso de interacción entre la institución y el entorno. En este sentido, la expresión de la extensión universitaria se dirige a la práctica, que según Pérez (2007, p. 94), se centra tradicional y normativamente en: 1) actividades de capacitación y adiestramiento, entendidas como educación continua, donde se concentra la visión de la generación de ingresos propios; 2) la promoción sociocultural y artística, donde, por tradición, hay una participación dinámica y activa por parte de las universidades; 3) acciones de trabajo con las comunidades donde generalmente se expresa que es poco lo realizado en este campo; y 4) las capacidades de la universidad para brindar asesoría al sector productivo, siendo un aspecto poco desarrollado y tratado dentro de las relaciones con el entorno.

Por consiguiente, la misión que tiene la universidad con su entorno es tan importante como la producción de conocimientos a través de la investigación, y la formación de profesionales competentes mediante la acción docente (Ferrer y Clemenza, 2009). Pero las actividades que se realizan actualmente en las universidades en pro de fortalecer su relación con el entorno, lucen rutinarias y descontextualizadas, con poco impacto en las necesidades que los nuevos tiempos le plantean tanto a la comunidad interna como externa. Al respecto, Canestrari (2001) ha señalado que la "universidad actual debe ser ventana abierta para recoger del entorno sus diversas manifestaciones, inquietudes y problemática y puente de salida de la cultura y de la ciencia para la transformación de la realidad circundante" (p. 55).

Ahora bien, la función de la universidad, en los albores del siglo XXI, recae en asumir los desafíos de la actualidad, así como estrechar y repensar la articulación con la sociedad (Villegas y Castillo, 2011). En correspondencia con lo planteado por Pérez (2007), en Venezuela la LSCEES da un giro a la extensión universitaria, intentando rescatar la relación directa universidadentorno al incorporar de manera masiva al estudiante a las actividades extramuros. Bajo estas consideraciones, la universidad se conecta con el entorno a través de la función de extensión y con apoyo del pregrado, conformando una estructura matricial entre la extensión, el servicio comunitario y el pregrado.

\section{El servicio comunitario en $L U Z$}

La Universidad del Zulia, siguiendo principios y lineamientos establecidos en la Constitución (1999), la Ley de Universidades (1970) y la LSCEES (2005), aprobó el Reglamento de Servicio Comunitario del Estudiante de Pregrado (2006), que sentó las bases para normar la prestación del servicio comunitario de los estudiantes de esta casa de estudios que aspiren al ejercicio de cualquier profesión, aplicando los conoci- 
mientos adquiridos durante su formación académica-profesional en beneficio de las comunidades.

Para el año 2012, este reglamento presentó una revisión y modificación en atención a la gestión académica-administrativa desarrollada hasta la fecha, que permitió crear la Comisión Central de Servicio Comunitario, que, junto con las coordinaciones de las facultades y núcleos, pasan a ser los entes administrativos encargados de la planificación, ejecución, control y evaluación de esta actividad académica, responsabilidades que anteriormente recaían sobre las facultades y núcleos en correspondencia con la Coordinación Central de Extensión de LUZ.

En esta reciente versión, la Comisión Central de Servicio Comunitario estará en coordinación con el Consejo Central de Extensión y Consejo Central de Pregrado de la universidad, conformando una tríada de principios y valores que reorganizan el modelo educativo docencia-servicio que, en esencia, es la síntesis dialéctica entre el saber y el hacer, promoviendo, así, la conexión universidad-entorno por medio de actores e instancias clave. De esta forma, se establece una estructura de tipo matricial en la cual el servicio comunitario funciona como eje transversal y está adscrito al Vicerrectorado Académico en el marco de la estructura organizativa de LUZ.

Los objetivos del servicio comunitario, de acuerdo con el artículo 5 del reglamento (2012), son: realizar el servicio mediante actividades enmarcadas en las políticas públicas, áreas estratégicas, planes, programas, subprogramas y proyectos de alto contenido e impacto social generados por la universidad, instituciones públicas y privadas, asociaciones gremiales y las comunidades; desarrollar en el estudiante competencias centradas en el ser, hacer, conocer y convivir; así como vincular la universidad con la comunidad, integrando saberes e intereses con justicia y compromiso social.
También son objetivos fortalecer la integración entre la docencia, la investigación y la extensión mediante la ampliación del conocimiento como beneficio socio-educativo hacia las comunidades; ampliar vínculos con la comunidad a través de una metodología socio-educativa del aprendizaje-servicio, aplicando el conocimiento adquirido durante la formación académica, artística, cultural y deportiva, que posibilite respuestas a las demandas del entorno social; además, promover y facilitar la formación integral del estudiante; $y$, finalmente, fomentar el desarrollo de proyectos que faciliten la participación de comunidades indígenas, afrodescendientes, discapacitados y desplazados.

De esta manera, el servicio comunitario le otorga al estudiante la oportunidad de interactuar con las comunidades, dejando un aporte significativo desde las diferentes áreas del conocimiento a través de las competencias desarrolladas durante su formación. Por este medio, se propicia la solidaridad estudiantil e institucional, la responsabilidad social, la participación protagónica, la cooperación y la corresponsabilidad con el desarrollo de las comunidades, incorporando la formación de valores humanos en el estudiante.

El artículo 3 del reglamento señala que el servicio es una actividad académica que debe ser concebida bajo la perspectiva del currículo integral, insertándose en los planes de estudio como eje transversal en concordancia con el Acuerdo $\mathrm{N}^{0} 535$ del Consejo Universitario (2006), desarrollando en el estudiante competencias cognoscitivas, procedimentales y actitudinales de tipo socio-comunitario. Asimismo, el referido reglamento expone, en los artículos 8 y 9, que esta actividad la realizará el estudiante que ha cumplido con el cincuenta por ciento (50\%) del total de la carga académica de sus estudios, con una duración de 120 horas académicas en total, distribuidas en un lapso no menor de tres (3) meses. 
Del mismo modo, el reglamento considera el tema de los recursos financieros y humanos para el avance de esta política institucional, siendo estos recursos los elementos clave para alcanzar los fines propuestos por el servicio. Puede decirse, siguiendo a Carrión (2007) y Robles (2012), que los recursos financieros están referidos a la disponibilidad económica de la organización, permitiendo hacer frente a compromisos que garanticen los medios para la consecución de sus objetivos. Por su parte, el recurso humano, de acuerdo a Rodríguez (2004) y Chiavenato (2007), es el factor crucial e insustituible para ejecutar las tareas y actividades de las organizaciones.

Bajo estos aspectos, el servicio comunitario en LUZ tiene como fuentes de financiamiento, según el artículo 41 del reglamento, los recursos asignados en el presupuesto ordinario de la universidad, financiamiento externo a través de convenios regionales, nacionales e internacionales y recursos derivados de procesos de autogestión, cogestión y promoción social.

En lo que respecta al recurso humano, el reglamento contempla que los actores clave del proceso son: el Coordinador de la Comisión Central, los coordinadores de las facultades y núcleos, los profesores-tutores y los estudiantes. El referido marco normativo puntualiza que las atribuciones de estos actores son velar por el cumplimiento de esta actividad académica y, para el caso específico de los coordinadores, dar cuenta ante el Consejo de Facultad y Consejo Universitario de los resultados e impactos de los proyectos ejecutados y representar a la universidad en actividades y reuniones de servicio comunitario, tanto a lo interno de la institución como a lo externo.

En tal sentido, LUZ ha realizado esfuerzos para institucionalizar esta política en el recinto universitario pues, desde el año 2005, se ha propuesto cumplir con las exigencias de la LSCEES, revisar el re- glamento interno en función de los resultados obtenidos y evaluar la pertinencia e impacto de los proyectos comunitarios ejecutados. No obstante, se hace necesario conocer la dinámica real de esta actividad que toma relevancia con el pasar del tiempo, específicamente en materia de recursos financieros y humanos, pues son los garantes de la ejecución de los objetivos académicos y administrativos del servicio.

\section{Recursos financieros y humanos en el servicio comunitario de LUZ: algunos resultados}

Como resultados, al aplicar las entrevistas, se obtiene, en referencia a los recursos financieros, que estos provienen principalmente de los recursos asignados al presupuesto ordinario de la universidad por parte del gobierno nacional. Al ingresar a las arcas de esta casa de estudios, se van a las facultades y núcleos para el funcionamiento de las actividades administrativas del servicio comunitario. Esta repartición se hace de acuerdo con el tamaño de la facultad en términos de población estudiantil.

El Coordinador de la Comisión Central de Servicio Comunitario manifiesta tener un limitado control de estos recursos. De esta manera, a pesar de que el reglamento (2012) le confiere la función de velar por el cumplimiento de la ejecución presupuestaria para las actividades del servicio comunitario y administrar tal presupuesto, la dinámica real muestra un matiz distinto. De hecho, la Coordinación de la Comisión Central actualmente carece de una estructura física administrativa-funcional para el desempeño de sus labores, sin embargo, desde sus espacios académicos, en la facultad de adscripción, cumple con sus compromisos.

Los coordinadores por facultades coincidieron que tampoco tienen control de los recursos, ya que estos ingresan directamen- 
te a la administración de las facultades y son estas instancias, junto con los decanos, quienes administran tales recursos. La administración de la facultad es quien suministra los materiales y equipos para el funcionamiento administrativo del servicio comunitario (computadoras, impresoras, materiales de oficina). Los coordinadores plantearon que sólo justifican trimestralmente a las unidades de planificación de las facultades las actividades realizadas y los impactos generados.

Ahora bien, el reglamento (2012), en el artículo 24, precisa las funciones de los coordinadores de servicios comunitarios de las facultades y núcleos y les delega la responsabilidad de preparar y presentar ante la Comisión Central de Servicio Comunitario el anteproyecto de presupuesto requerido para cumplir con las actividades de servicio comunitario. Tal responsabilidad, en la dinámica real, recae sobre las unidades de planificación de las facultades. Los coordinadores del servicio sólo gestionan las actividades académicas de los tutores y los estudiantes en las comunidades.

Cabe señalar que, en algún momento, los recursos asignados para el servicio alcanzaron para adquirir equipos (computador, impresora, escáner) y materiales de oficina suficientes para las dependencias encargadas de esta actividad, pero, en la actualidad, la misma situación inflacionaria del país ha traído problemas para obtener lo esencial del trabajo administrativo. Cada decano ha tenido que jugar estratégicamente con el resto de las partidas presupuestarias o ingresos propios de la facultad para atender las demandas que hacen las dependencias y no descuidar esta actividad académica.

En diversas oportunidades se han tenido que buscar recursos por cogestión y promoción social con organizaciones extrauniversitarias, pero estos aportes representan una mínima parte de lo requerido, sin embargo, han ayudado para el desarrollo de alguna actividad en particular. Los recursos que se obtienen por esta vía los han tramitado los tutores en compañía de sus estudiantes, dependiendo de la comunidad o instancia pública o privada a atender y que desean apoyar con el servicio comunitario bajo una relación ganar-ganar. Situación que ocurre en otras instituciones de educación superior, de acuerdo con Urdaneta et al., (2014).

Los informantes clave también señalan que la mayoría de las veces se recurre a la autogestión o autofinanciamiento, el cual proviene, principalmente, de los aportes económicos de los estudiantes y profesorestutores. A manera de ejemplo señalan que, tanto estudiantes como profesores, aportan dinero para comprar materiales, refrigerios, entre otros insumos; asimismo, colocan sus vehículos a disposición para el traslado a la comunidad donde se ejecuta el proyecto. La situación descrita permite percibir ciertas limitaciones financiaras para llevar a cabo el servicio comunitario, lo que puede incidir en la desmotivación de estudiantes y tutores.

En cuanto al recurso humano disponible para esta actividad, como ya se mencionó, se cuenta principalmente con el Coordinador de la Comisión Central de Servicio Comunitario, los coordinadores por facultades, los profesores-tutores y los estudiantes. Además, las coordinaciones de las facultades estudiadas poseen coordinadores por escuelas y un personal administrativo de apoyo. Todos conforman el recurso humano garante de desarrollar el servicio comunitario, con previo estudio de las necesidades y demandas sociales.

A inicios de la política del servicio, los universitarios se resistían a insertarse en este nuevo paradigma, que era visto como una imposición con tinte político por parte del gobierno nacional. En palabras de Ferrer y Clemenza (2009, p. 119), "existía una re- 
sistencia marcada al desarrollo de estas actividades". Con el pasar del tiempo, y a través de las revisiones de las actividades ejecutadas, el recurso humano ha entendido la dinámica del mismo y se ha mostrado proactivo en colaborar para realizar las distintas actividades planificadas por las escuelas de cada facultad en apoyo a un determinado sector.

Los coordinadores de las facultades concuerdan en que, en la actualidad, los tutores se sienten identificados con sus proyectos, pues las propuestas para atender a una comunidad en específico o alguna instancia pública provienen de ellos mismos. De este modo, refieren que los tutores en su práctica docente entusiasman a sus estudiantes e inmediatamente los vinculan a su proyecto.

De igual manera, comentan que los estudiantes entran al servicio con resistencia y desmotivación, pues deben sacrificar parte de su tiempo en un dinamismo casi desconocido para ellos. No obstante, relatan que al final de la actividad aprecian otra actitud en los universitarios, ya que estos pudieron contribuir en la solución de algún problema de una comunidad, aplicando los sabes adquiridos hasta ese momento en su formación académica-profesional.

Finalmente, los entrevistados exhortaron a las autoridades de la universidad a promover con campañas de sensibilización las actividades del servicio comunitario para que los actores del proceso se sientan más identificados y comprometidos. Asimismo, invitaron a los profesores de las diferentes escuelas a sumarse con proyectos de interés y pertinencia para la comunidad de la región. A los estudiantes les invitan a cumplir y disfrutar este acercamiento entre la universidad y el entorno, donde activan las competencias de ser, hacer, conocer y convivir en la práctica de los conocimientos obtenidos en su carrera.

\section{Conclusiones}

El servicio comunitario es una actividad que no puede confundirse con labor social, altruismo o regalía; comprende una metodología de trabajo y un proceso que conecta la extensión universitaria con el pregrado, de donde emergen las fuerzas de vinculación universidad-entorno con protagonismo estudiantil, pues esta tarea está a cargo de los futuros profesionales del país, quienes tienen la oportunidad de ofrecer aportes al entorno social, aplicando los conocimientos adquiridos o las competencias desarrolladas hasta ese momento en su formación.

Esta política nacional ha trascendido con el pasar del tiempo y se ha fortalecido producto de las revisiones que se le han hecho a las actividades ejecutadas. Desde las universidades se ha emprendido en mejorar la metodología aprendizaje-servicio, que es garante de alcanzar resultados positivos para las comunidades. La Universidad del Zulia se ha esforzado también en dar respuesta, a través del servicio, a las demandas que hace el entorno; existen un sin número de proyectos en las diferentes parroquias de los municipios del estado Zulia, en instituciones públicas, privadas o de carácter social con alto impacto.

El tema de los recursos financieros se ha convertido en la variable que ha llamado la atención de los gestores del proceso en la universidad, ya que la situación económica que vive el país ha arropado la dinámica de estas actividades en términos académicos y hasta administrativos, teniéndose que optar por la autogestión, la cual puede estar incidiendo en el desestímulo de algunos actores clave, como el caso específico de tutores y estudiantes. Los recursos provenientes del presupuesto ordinario lo tienen centralizado prácticamente las administraciones de las facultades. No obstante, se realizan gestiones para no desatender los requerimientos básicos del servicio. 
Por estas razones, se hace necesario elevar ante las instancias gubernamentales y líderes de esta política la revisión del presupuesto asignado para que éste sea suficiente y digno para ejecutar las iniciativas de la universidad con la comunidad, puesto que se cuenta con el recurso humano suficiente (que además está dispuesto a colaborar), pero no tiene los insumos suficientes para generar proyectos de alta pertinencia. Se está a tiempo de seguir reconociendo debilidades para revertirlas en fortalezas y oportunidades para la integración sincera de la universidad con el entorno.

\section{Referencias}

Asamblea Nacional Constituyente. (1999). Constitución de la República Bolivariana de Venezuela. Gaceta Oficial $\mathrm{N}^{\circ}$ 5.908. Venezuela.

Asamblea Nacional de la República Bolivariana de Venezuela. (2005). Ley de Servicio Comunitario del Estudiante de Educación Superior. Gaceta Oficial $\mathrm{N}^{\mathrm{o}}$ 38.272. Venezuela.

Canestrari, M. (2001). Reflexiones sobre la transformación de la UCV. Debate Abierto, Revista Venezolana para la Reflexión y Discusión, Ciencias Científicas Venezolanas de la AsoVAC (Asociación Venezolana para el Avance de la Ciencia), año V, vol. IV, 50-56. Caracas, Venezuela.

Carrión, J. (2007). Estrategia. España: Editorial Esic.

Chiavenato, I. (2007). Administración de recursos humanos. El capital humano de las organizaciones. Octava edición. España: McGRAW-HILL/INTERAMERICANA.

Congreso de la República de Venezuela. (1970). Ley de Universidades. Gaceta Oficial Extraordinaria $\mathrm{N}^{\mathrm{o}} 1429$. Venezuela.
Consejo Universitario. (2006). Acuerdo $N^{o}$ 535. Universidad del Zulia, Venezuela.

Consejo Universitario. (2006). Reglamento de Servicio Comunitario del Estudiante de Pregrado en la Universidad del Zulia. Derogado en el 2012. Universidad del Zulia, Venezuela.

Consejo Universitario. (2012). Reglamento de Servicio Comunitario del Estudiante de Pregrado en la Universidad del Zulia. Universidad del Zulia, Venezuela.

Ferrer, J. y Clemenza, C. (2009). Servicio comunitario del estudiante en el contexto de la educación superior venezolana. Revista Venezolana de Gerencia, $R V G$, 14(45), 110 - 124. Maracaibo, Venezuela.

Hernández, D. (2006). La Responsabilidad Social Universitaria y la Ley de Servicio Comunitario del Estudiante de Educación Superior. Guevara, J. (Ed.), Estudios sobre la nueva Ley de Servicio Comunitario del Estudiante de Educación Superior (pp. 51-62). Venezuela: Ediciones Paredes.

Malagón, L. (Noviembre, 2002). Pertinencia y Educación Superior. Una mirada crítica. Tercer Congreso Nacional y Segundo Internacional "Retos y Expectativas de la Universidad", UNAM, México.

Pérez, F., Barrios, M., Brizuela, B., Gómez, L., Gutiérrez, C., Mariño, A., Mariño, M., Palencia, O., Pinto, F. y Pinto, E. (Enero-abril de 2014). La participación social y el servicio comunitario del nivel de educacion superior: Una hermeneusis desde la perspectiva de su ejecución. Nexos, Revista Electrónica de Investigación y Postgrado, 3(1), 9 - 17. Guárico, Venezuela. 
Pérez, T. (2007). Caracterización de los vínculos de la extensión universitaria con las carreras de educación integral de la UNA. Un enfoque descriptivo interpretativo e iluminativo. Venezuela: Fondo editorial IPASME, Universidad Nacional Abierta.

Ramos, C. (2007). Universidades, cultura innovativa y vinculación con el entorno socioproductivo. Venezuela: Ediciones del Vicerrectorado Académico, Universidad del Zulia.

Robles, C. (2012). Fundamentos de administración financiera. México: Editorial Red Tercer Milenio.

Rodríguez, J. (2004). El modelo de gestión de recursos humanos. España: Editorial UOC.

Torres, P. y Cano, C. (1997). Informe de los resultados. I Jornadas de Extensión Universitaria del Estado Guárico. Venezuela: Universidad Rómulo Gallegos y Fundación Ciara.
Tünnerman, C. (2000). Universidad y Sociedad. Balance Histórico desde Latinoamérica. Venezuela: Ministerio de Educación, Cultura y Deportes.

Tünnerman, C. (2003). La universidad latinoamericana ante los retos del siglo XXI. México: Unión de Universidades de América Latina.

Urdaneta, E., Godoy, M y Barreto, M (2014). El servicio comunitario desde la perspectiva del estudiante del Núcleo Universitario Rafael Rangel de la Universidad de los Andes, Trujillo. Revista ACADEMIA, 13(32), 53-63. Trujillo, Venezuela.

Villegas, D. y Castillo, N. (2011). La responsabilidad social y el servicio comunitario en la educación universitaria. Revista Científica Digital del Centro de Investigación y Estudios Gerenciales, Rcieg, 1(7), 94-110. Barquisimeto, Venezuela. 